\title{
Radioembolization versus portal vein embolization for contralateral liver lobe hypertrophy: effect of cirrhosis
}

\author{
Heiner Nebelung ${ }^{1} \odot$ - Thomas Wolf ${ }^{1} \cdot$ Sebastian Bund ${ }^{1} \cdot$ Christoph Georg Radosa ${ }^{1}$. Verena Plodeck ${ }^{1}$. \\ Sabine Grosche-Schlee ${ }^{2}$. Carina Riediger ${ }^{3} \cdot$ Ralf-Thorsten Hoffmann ${ }^{1} \cdot$ Jens-Peter Kühn ${ }^{1}$
}

Received: 30 January 2021 / Revised: 3 March 2021 / Accepted: 9 March 2021 / Published online: 29 March 2021

(c) The Author(s) 2021

\begin{abstract}
Purpose Preoperative hypertrophy induction of future liver remnant (FLR) reduces the risk of postoperative liver insufficiency after partial hepatectomy. One of the most commonly used methods to induce hypertrophy of FLR is portal vein embolization (PVE). Recent studies have shown that transarterial radioembolization (TARE) also induces hypertrophy of the contralateral liver lobe. The aim of our study was to evaluate contralateral hypertrophy after TARE versus after PVE taking into account the effect of cirrhosis.

Methods Forty-nine patients undergoing PVE before hemihepatectomy and 24 patients with TARE as palliative treatment for liver malignancy were retrospectively included. Semi-automated volumetry of the FLR/contralateral liver lobe before and after intervention (20 to 65 days) was performed on CT or MRI, and the relative increase in volume was calculated. Cirrhosis was evaluated independently by two radiologists on CT/MRI, and interrater reliability was calculated.

Results Hypertrophy after PVE was significantly more pronounced than after TARE $(25.3 \%$ vs. $7.4 \% ; p<0.001)$. In the subgroup of patients without cirrhosis, the difference was also statistically significant ( $25.9 \%$ vs. $8.6 \%$; $p=0.002)$, whereas in patients with cirrhosis, the difference was not statistically significant (18.2\% vs. $7.4 \% ; p=0.212)$. After PVE, hypertrophy in patients without cirrhosis was more pronounced than in patients with cirrhosis $(25.9 \%$ vs. $18.2 \% ; p=0.203)$, while after TARE, hypertrophy was comparable in patients with and without cirrhosis (7.4\% vs. 8.6\%; $p=0.928)$.

Conclusion TARE induces less pronounced hypertrophy of the FLR compared to PVE. Cirrhosis seems to be less of a limiting factor for hypertrophy after TARE, compared to PVE.
\end{abstract}

Heiner Nebelung

heiner.nebelung@uniklinikum-dresden.de

1 Department of Radiology, Dresden University Hospital, Fetscherstr. 74, 01307 Dresden, Germany

2 Department of Nuclear Medicine, Dresden University Hospital, Fetscherstr. 74, 01307 Dresden, Germany

3 Department of Visceral, Thoracic and Vascular Surgery, Dresden University Hospital, Fetscherstr. 74, 01307 Dresden, Germany 


\section{Graphic abstract}

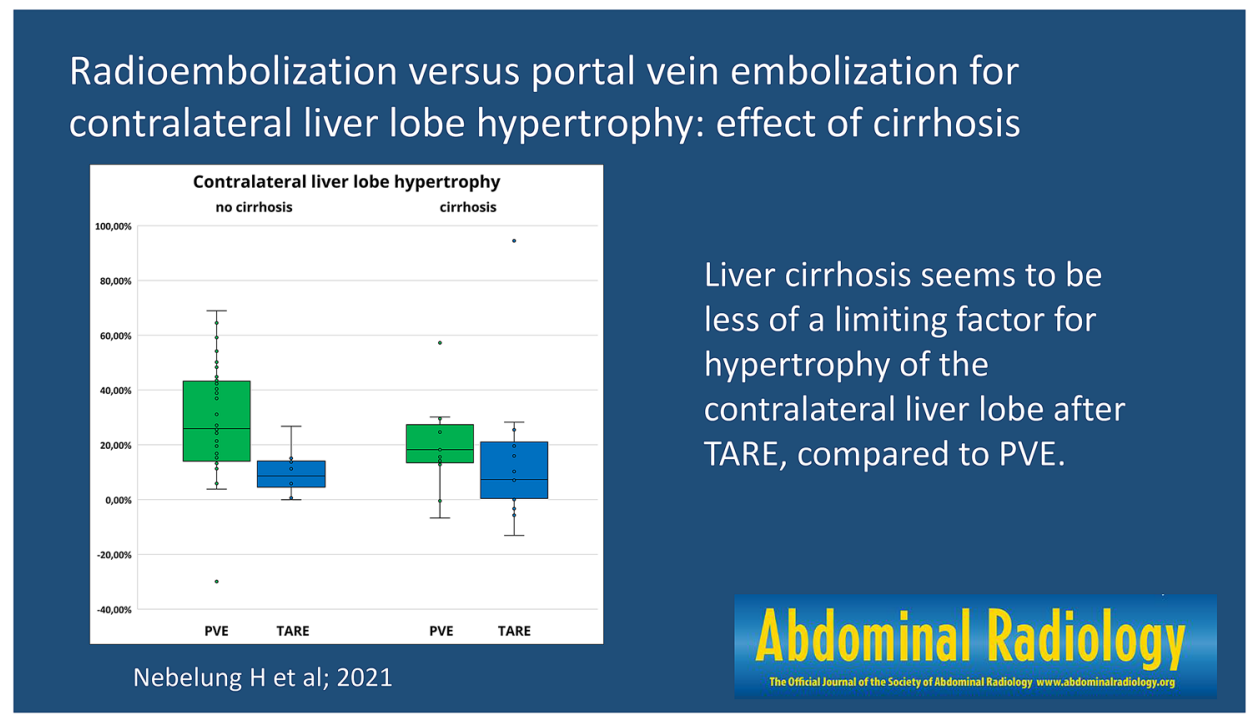

Keywords Therapeutic embolization $\cdot$ Hypertrophy $\cdot$ Liver cirrhosis $\cdot$ Portal vein $\cdot$ Hepatic artery

\section{Abbreviations}

FLR Future liver remnant

IQR Interquartile range

PVE Portal vein embolization

TARE Transarterial radioembolization

\section{Introduction}

Partial hepatectomy is frequently the curative treatment of choice for malignant tumors of the liver. Depending on tumor size, location, and number of lesions, extensive resections up to extended hemihepatectomy can be necessary. Resection of more than $75 \%$ of hepatic parenchyma more than triples the risk of postoperative hepatic dysfunction [1]. Preoperative hypertrophy induction of the future liver remnant (FLR) can lead to an increase in functioning liver parenchyma and, therefore, reduces the risk of postoperative liver insufficiency. The currently best-studied methods to induce hypertrophy of FLR are portal vein embolization (PVE) and portal vein ligation [2,3], which is often combined with partition of liver parenchyma ("in situ split") [4]. Recent studies have shown that transarterial radioembolization (TARE) also induces hypertrophy of the contralateral, untreated liver lobe [5-7].

Physiologically, total hepatic blood flow consists of $70-80 \%$ portal venous and $20-30 \%$ hepatic arterial blood. In patients with cirrhosis, portal blood flow decreases and the "hepatic arterial buffer response" keeps total hepatic flow at a constant level by increasing hepatic arterial blood flow [8].
Occlusion of portal veins of the liver segments that will be resected leads to redirection of portal blood to the FLR. This way, PVE leads to hypertrophy of the untreated liver segments [9]. In general, there is high variability in hypertrophy after PVE. Many influencing factors have been discussed in the literature, e.g., intrahepatic tumor burden [10], hyperbilirubinemia [11], and BMI [12], but overall the physiology of liver hypertrophy is not well understood. In patients with cirrhosis, sufficient hypertrophy seems to take longer than in patients without cirrhosis [13]. A disadvantage of PVE is possible tumor progress during hypertrophy induction, since malignant tumors recruit their blood supply from arterial vessels [14].

TARE is currently used as a palliative treatment for malignant liver diseases. It allows for selective radiation therapy of hypervascular lesions [15]. As a result, local tumor control and downsizing can be achieved, possibly leading to resectability of the lesions. Several studies have shown that PVE induces more pronounced contralateral hypertrophy [6] and TARE-induced hypertrophy of the untreated liver lobe seems to take longer than after PVE [5], but there is little data on the effect of cirrhosis on hypertrophy after TARE. One study by Teo et al. showed a significantly greater degree of hypertrophy after TARE in HCC patients with hepatitis $\mathrm{B}$, compared to those with hepatitis $\mathrm{C}$ or alcoholic cirrhosis [7]. Another study by Edeline et al. stated that TARE induces similar increases in FLR volume compared to PVE in patients with cirrhosis [16]. A recent review by Birgin et al. analyzed a total of 16 studies, comprising 602 patients, and stated that the influence of cirrhosis on hypertrophy of 
the contralateral liver lobe after TARE remains controversial, with no significant difference of kinetic growth rate in the three included studies which assessed only patients with cirrhosis, compared to patients without cirrhosis [17].

Thus, the aim of our study was to evaluate the effect of cirrhosis on contralateral liver lobe hypertrophy after TARE versus after PVE.

\section{Materials and methods}

\section{Study population and indications}

This study was approved by the local ethics committee. Due to the retrospective evaluation, written informed consent was waived. The radiology information system of our hospital was searched for patients who underwent PVE before hemihepatectomy in curative intention between August 2015 and December 2019 and patients who underwent TARE as a palliative treatment for malignant liver diseases between October 2013 and February 2019. Inclusion criteria were intervention of the right liver lobe ( \pm segment IV), preinterventional CT or MRI, and postinterventional CT or MRI between 20 and 65 days after TARE or PVE. Postinterventional imaging was performed prior to surgery after PVE and along in-hospital follow-up protocols after TARE. Exclusion criteria were preinterventional partial liver resections, preinterventional in situ split, a prior transarterial chemoembolization or prior PVE. Some patients were excluded because they underwent resection or second interventional treatment without intermediary follow-up. Further exclusion criteria were incomplete PVE in the PVE group and treatment with embolization agents other than Yttrium-90 in the TARE group. Seventy-three patients fulfilled all inclusion criteria, including 49 patients who underwent PVE and 24 patients who underwent TARE (Fig. 1; Table 1).

\section{Technique of interventions}

All interventions were performed on an image-guided therapy system (Philips Azurion 7 M20, Philips Healthcare).

$P V E$ After ultrasound-guided (Philips Affiniti 70G, Philips Healthcare) puncture (chiba needle, Peter Pflugbeil $\mathrm{GmbH}$ ) of a right portal vein branch, a 5F sheath (DilPlus, Peter Pflugbeil $\mathrm{GmbH}$ ) is introduced and the main portal vein accessed via a guidewire (Nitrex Guidewire, Medtronic). Using a Simmons catheter (SIM-catheter, Cardinal Health), a diagnostic portogram is performed to assess portal vein anatomy. After placing the Simmons catheter in the right portal vein, a microcatheter (Progreat, Terumo $\mathrm{GmbH}$ ) is then placed in the anterior and posterior pedicle of the right portal vein (in some cases also the portal vein branch supplying segment IV) and embolization is performed using a 1:5 mixture of histoacryl (Histoacryl ${ }^{\circledR}, \mathrm{B}$. Braun Melsungen AG) and lipiodol (Lipiodol@ Ultra-Fluid, Guerbet $\mathrm{GmbH}$ ).

TARE After puncture (puncture needle, Cardinal Health) of the common femoral artery, introduction of a $5 \mathrm{~F}$ sheath (Glidesheath Slender, Terumo Deutschland $\mathrm{GmbH}$ ), and cannulation of the celiac trunk (Cobra-catheter, Cardinal Health), diagnostic angiography is performed. Collaterals,
Fig. 1 Study population. After applying exclusion criteria, 49 of 118 patients who underwent PVE from $08 / 2015$ to $12 / 2019$ and 24 of 153 patients who underwent TARE from 10/2013 to $02 / 2019$ were included
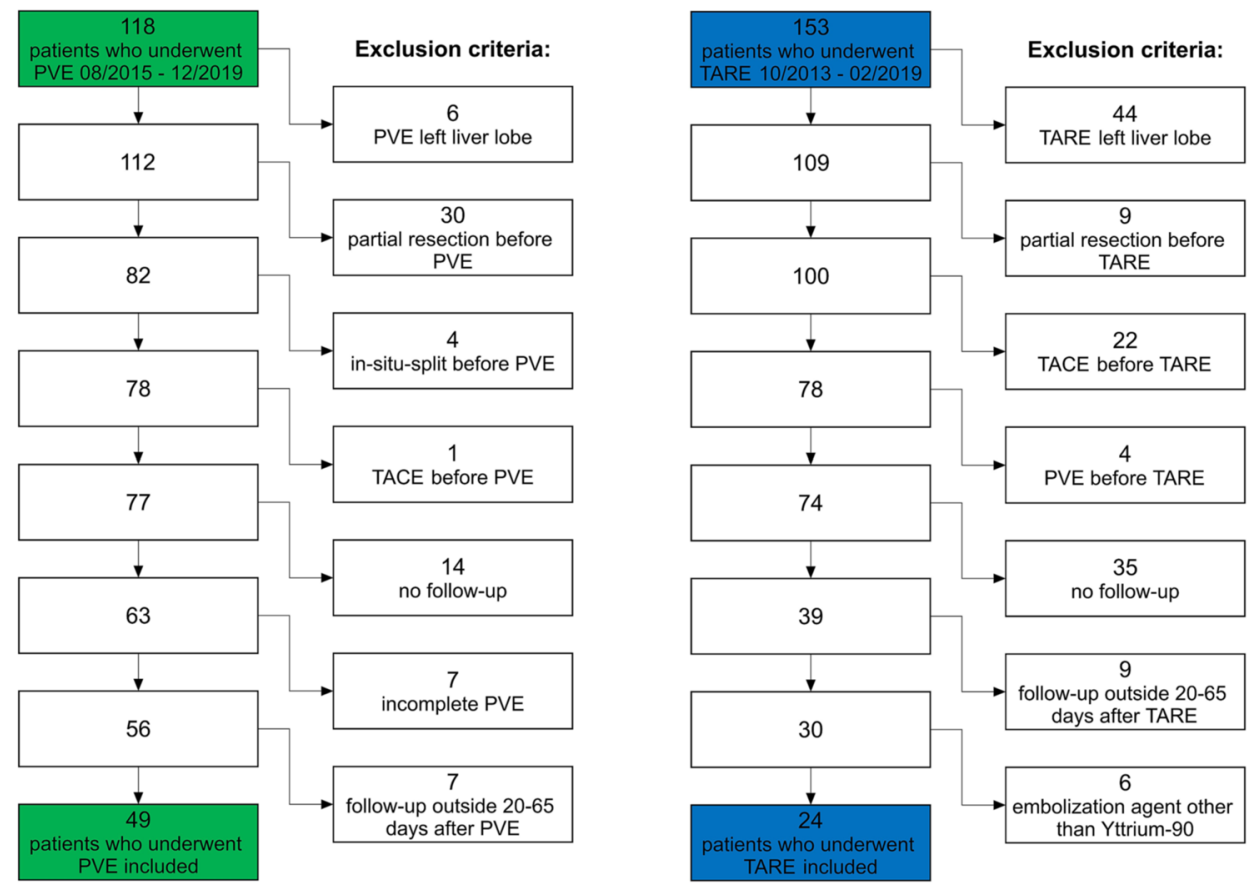


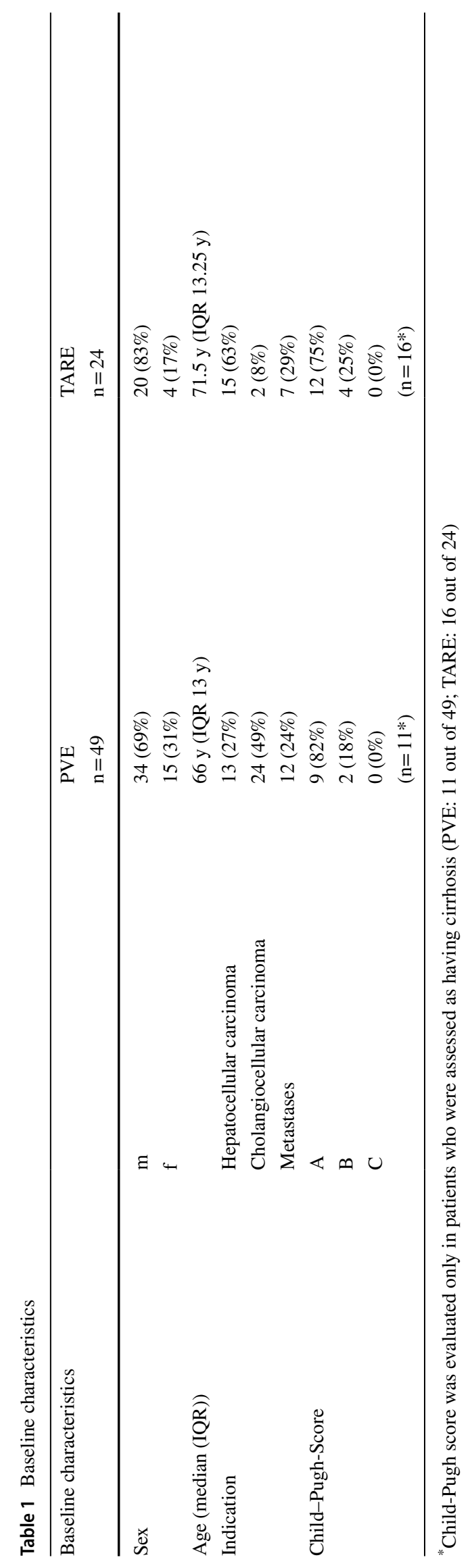


Fig. 2 CT scans before (left) and about two months after PVE (right) with volumetry of the FLR (blue)
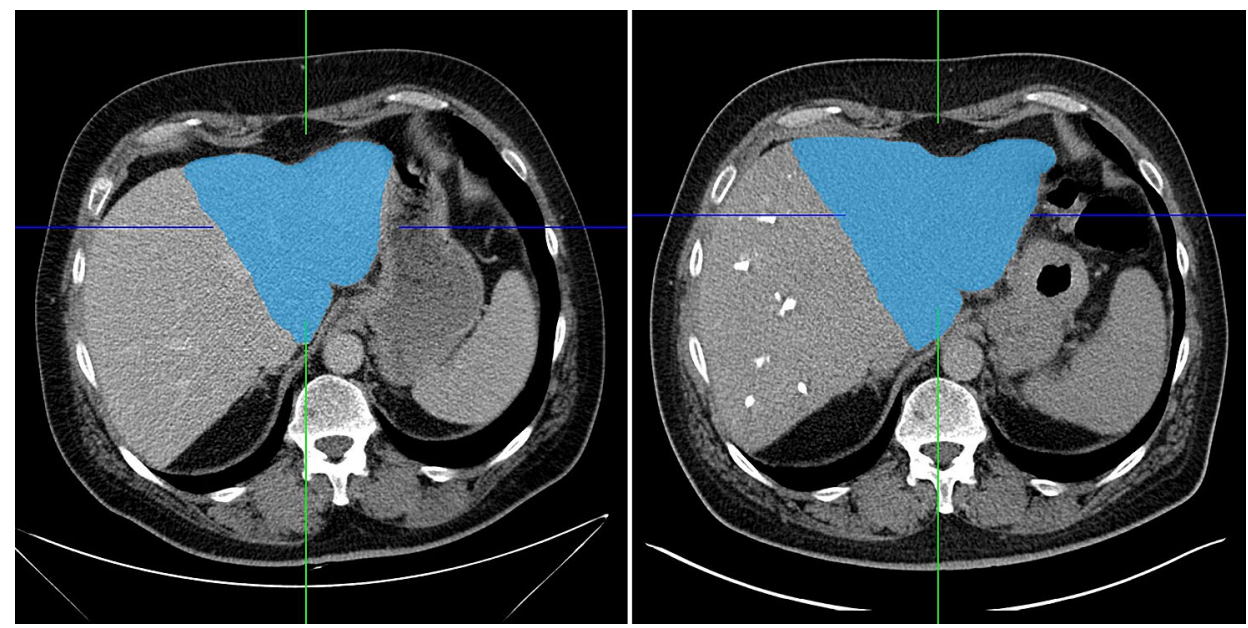

Fig. 3 CT scans before (left) and about two months after TARE (right) with volumetry of the contralateral liver lobe (blue)
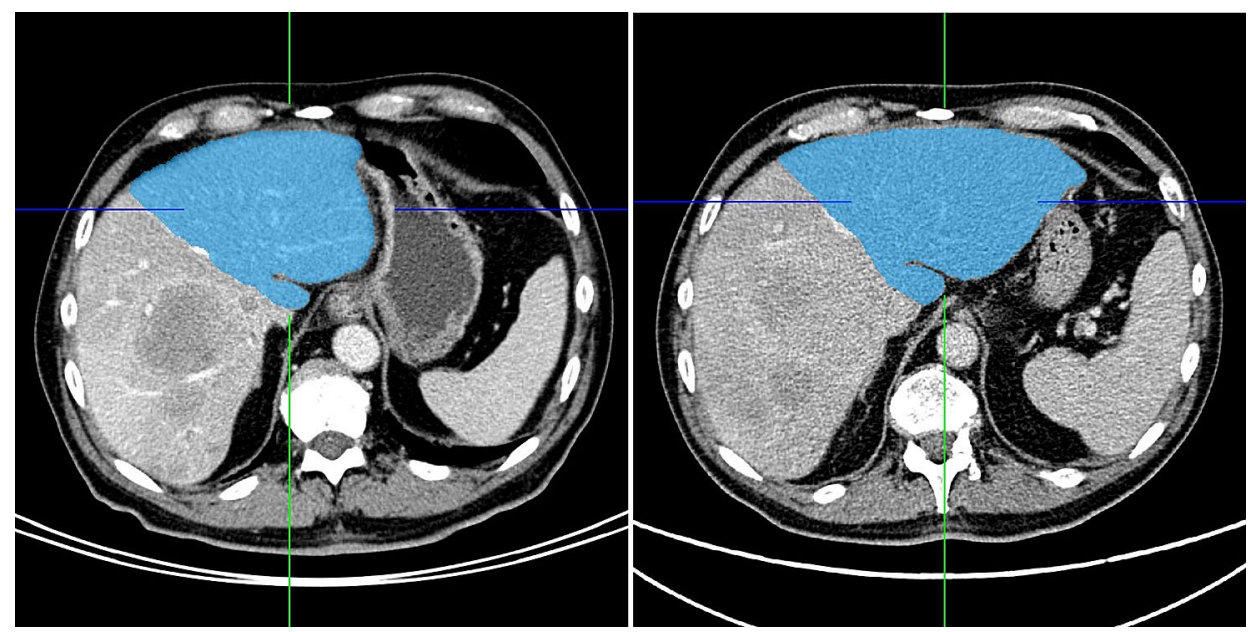

which might lead to unwanted spread of the radioembolization agent into other organs (mainly via the gastroduodenal artery), are identified and occluded using coil embolization where appropriate. The right hepatic artery is cannulated using a microcatheter (Progreat, Terumo GmbH). Technetium-99m-HSA microspheres (ROTOP HSA Mikrosphären B20, ROTOP) are applied to assess the distribution. In a second session, Yttrium-90 microspheres (SIR-Spheres Resin Microspheres, Sirtex Medical) are applied selectively into the right hepatic artery. To estimate each individual dosage of Yttrium-90 microspheres for SIRT, several technical and clinical considerations are taken into account, as described previously by Salem et al. [18]. Using pretreatment diagnostic CT or MR imaging, diagnostic angiography and Technetium-99m-MAA SPECT/CT scanning, activities are calculated according to the body surface area method and/or the partition model, if applicable [19]. Thus, therapy dosages are adjusted in respect to the tumor mass, the uninvolved liver parenchyma, the lung shunt fraction, and the patient's clinical status, respectively. Planned dosages are reduced up to $20 \%$ in patients with pre-existing liver damage to prevent radioembolization-induced liver disease [20]. In our study, median administered activity for patients undergoing TARE was $1100 \mathrm{MBq}$ (IQR $1670 \mathrm{MBq}$ ).

\section{Evaluation of cirrhosis}

Cirrhosis (present/not present) was evaluated independently by two radiologists on CT/MRI (one radiologist with more than 15 years of experience in liver imaging, one resident with 2 years of experience in liver imaging). They were blinded to the type of intervention (TARE or PVE). They used the following criteria: hypertrophy of the caudate lobe and segments II/III with concomitant atrophy of segments VI/VII [21], surface and parenchymal nodularity and heterogeneity, portal vein enlargement, portal venous thrombosis, and ascites [22]. In case of disagreement, the result was decided in consensus. 
Fig. 4 Contralateral liver lobe hypertrophy in patients who underwent PVE and in patients who underwent TARE

\section{Contralateral liver lobe hypertrophy}

PVE

TARE

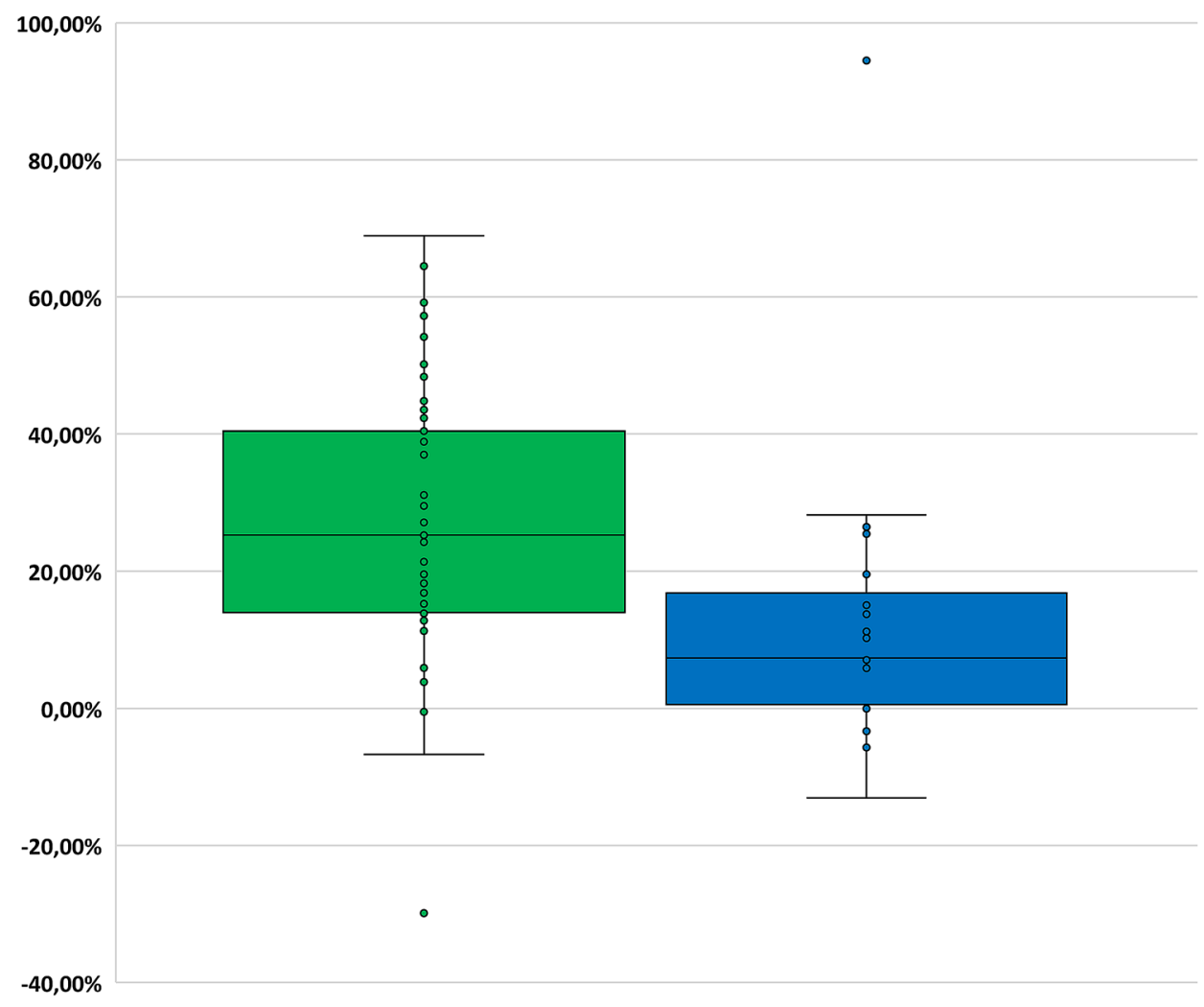

Table 2 Contralateral liver lobe hypertrophy in patients who underwent PVE and in patients who underwent TARE

\begin{tabular}{lll}
\hline & \multicolumn{2}{l}{$\begin{array}{l}\text { Contralateral liver lobe hypertrophy } \\
\text { Median (Interquartile range) }\end{array}$} \\
\cline { 2 - 3 } & Hypertrophy & Time interval \\
\hline PVE & $25.3 \%(27.4 \%)$ & 33 days (16 days) \\
TARE & $7.4 \%(18.1 \%)$ & 52,5 days (16 days) \\
& $p<0.001$ & \\
\hline
\end{tabular}

\section{Volumetry}

Volumetry of the FLR before and after PVE or the contralateral liver lobe before and after TARE was performed using the contour segmentation tool with semi-automated edge detection of IMPAX (IMPAX EE R 20, Agfa HealthCare) by two independent raters. Both were trained prior to the evaluation by a radiologist with more than 15 years of experience in liver imaging (Figs. 2, 3).

\section{Statistics}

At first, all variables to be compared were examined for normal distribution by the Shapiro-Wilk test [23]. Since in most cases there was no normal distribution, medians and interquartile ranges (IQR) were calculated and non-parametric tests were used for all comparisons [24].

Relative increase in volume was calculated and compared using the Mann-Whitney $U$ test. The significance level was set at $\alpha=0.05$ in all cases. Time interval between intervention and postinterventional CT/MRI was documented.

Regarding the evaluation of cirrhosis, interrater reliability was evaluated using Cohens kappa $(\kappa)[25,26]$, which was interpreted according to the recommendations of Landis and Koch [27] ( $\kappa \leq 0$ poor; 0.01-0.20 slight, 0.21-0.40 fair, $0.41-0.60$ moderate, $0.61-0.80$ substantial and $0.81-1.00$ almost perfect agreement).

The Shapiro-Wilk test, Mann-Whitney $U$ test, and the calculation of Cohens kappa were performed using SPSS (IBM Corp. Released 2018. IBM SPSS Statistics for Windows, Version 26.0). Diagrams and tables were created using Microsoft Office Excel 2016 (Microsoft Corporation).

\section{Results}

Out of 49 patients who underwent PVE, 11 patients were assessed as having cirrhosis (22\%), 38 patients as not having cirrhosis (78\%). Out of 24 patients who underwent TARE, 
there were 16 patients with cirrhosis $(67 \%)$ and 8 patients without (33\%).

In general, hypertrophy after PVE was significantly more pronounced than after TARE (25.3\% (IQR 27.4\%) vs. 7.4\% (IQR $18.1 \%) ; p<0.001$ ), although time intervals between PVE and postinterventional CT/MRI were shorter than between TARE and postinterventional CT/MRI [33 days (IQR 16 days) vs. 52.5 days (IQR 16 days)] (Fig. 4; Table 2).

In the subgroup of patients without cirrhosis, hypertrophy after PVE was significantly more pronounced than after TARE [25.9\% (IQR 29.8\%) vs. 8.6\% (IQR 12.8\%); $p=0.002]$, whereas time intervals were similar to the overall study population [32 days (IQR 15 days) vs. 46 days (IQR 24 days)]. In the subgroup of patients with cirrhosis, hypertrophy after PVE was more pronounced than after TARE, but the difference was not statistically significant $[18.2 \%$ (IQR $16.7 \%$ ) vs. $7.4 \%$ (IQR 23.8\%); $p=0.212$ ]. Time intervals between PVE and postinterventional CT/MRI were shorter than between TARE and postinterventional CT/MRI [34 days (IQR 24 days) vs. 56 days (IQR 10 days)], similar to the overall study population (Fig. 5; Table 3).

After PVE, hypertrophy in patients without cirrhosis was more pronounced than in patients with cirrhosis [25.9\% (IQR 29.8\%) vs. $18.2 \%$ (IQR 16.7\%); $p=0.203$ ]. After TARE, hypertrophy was similar in patients with cirrhosis and without [7.4\% (IQR 23.8\%) vs. 8.6\% (IQR 12.8\%); $p=0.928]$ (Fig. 5; Table 3).
Fig. 5 Contralateral liver lobe hypertrophy in patients with cirrhosis and without (after PVE and after TARE)
Table 3 Contralateral liver lobe hypertrophy in patients with cirrhosis and without (after PVE and after TARE)

\section{Contralateral liver lobe hypertrophy \\ no cirrhosis \\ cirrhosis}
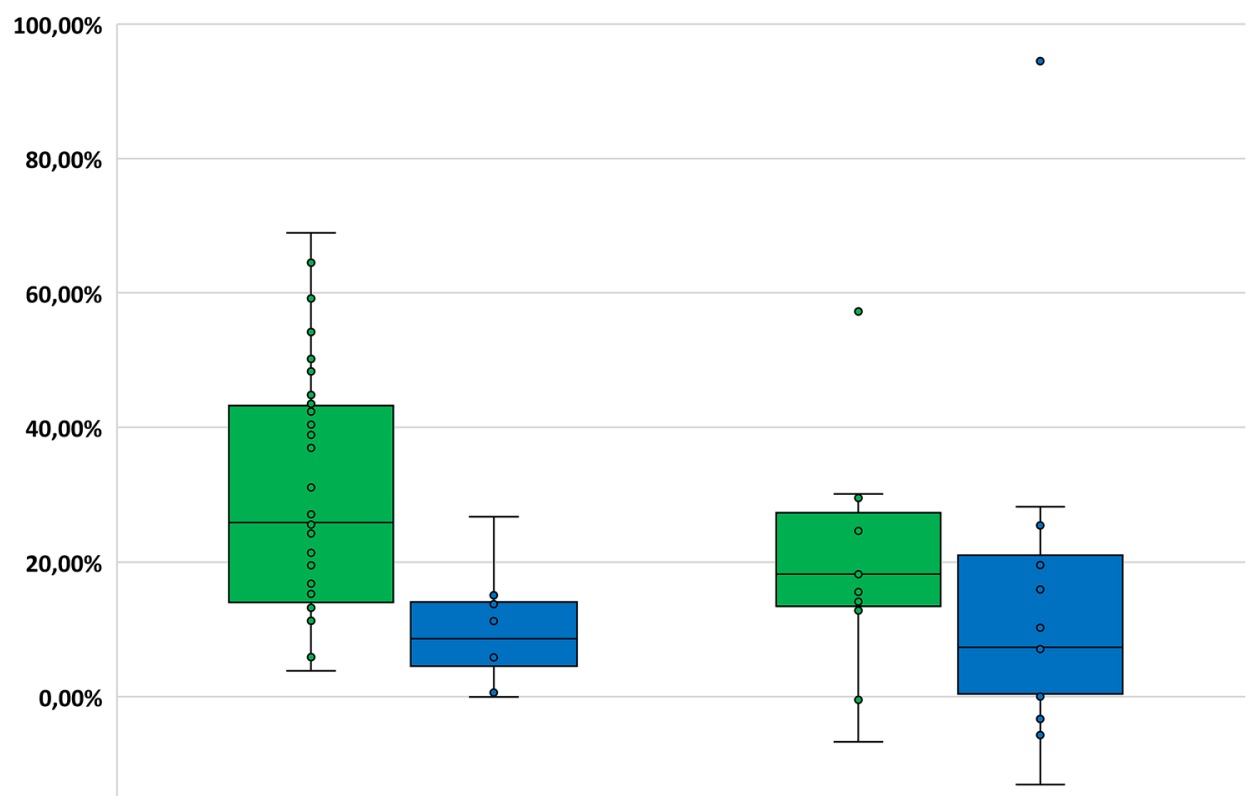

$-20,00 \%$

$-40,00 \%$

PV

TARE

PVE

TARE

\begin{tabular}{|c|c|c|c|c|c|}
\hline & \multicolumn{4}{|c|}{$\begin{array}{l}\text { Contralateral liver lobe hypertrophy } \\
\text { Median (Interquartile range) }\end{array}$} & \\
\hline & \multicolumn{2}{|l|}{ No cirrhosis } & \multicolumn{2}{|l|}{ Cirrhosis } & \\
\hline & Hypertrophy & Time interval & Hypertrophy & Time interval & \\
\hline PVE & $25.9 \%(29.8 \%)$ & 32 days (15 days) & $18.2 \%(16.7 \%)$ & 34 days (24 days) & $p=0.203$ \\
\hline \multirow[t]{2}{*}{ TARE } & $8.6 \%(12.8 \%)$ & 46 days ( 24 days) & $7.4 \%(23.8 \%)$ & 56 days (10 days) & $p=0.928$ \\
\hline & $p=0.002$ & & $p=0.212$ & & \\
\hline
\end{tabular}




\section{Interrater reliability}

For the evaluation of cirrhosis, both radiologists assigned identical scores in $87.7 \%$ (64/73) and different scores in $12.3 \%$ (9/73). The resulting Cohens kappa was $\kappa=0.741$, indicating substantial interrater agreement.

\section{Discussion}

Our study shows that PVE leads to significantly more pronounced hypertrophy of the FLR in a shorter time interval than TARE, particularly in patients without cirrhosis. These results are in keeping with recent studies, e.g., with a study by Garlipp et al. [6] and with studies by Teo et al. [5, 7]. A possible reason is the large contribution of portal blood flow on total hepatic perfusion and the resulting high impact of occluded portal veins.

In the subgroup of patients without cirrhosis, hypertrophy after PVE was also significantly more pronounced than after TARE, whereas in patients with cirrhosis, there was a similar trend, but this was not statistically significant. The most likely cause for the latter are the relatively low case numbers in these subgroups of the study, as the median for hypertrophy after PVE was still more than twice the median for hypertrophy after TARE.

After PVE, hypertrophy was more pronounced in patients without cirrhosis than in patients with cirrhosis, although the difference was not statistically significant. Indirectly, this result is in keeping with a study by Madoff et al. which showed that sufficient hypertrophy seems to take longer in patients with cirrhosis than in patients without cirrhosis [13]. In contrast, hypertrophy after TARE was similar in patients with cirrhosis and without. A possible explanation is the decreased portal blood flow caused by the cirrhosis and the compensatory higher proportion of hepatic arterial blood flow in regard to total hepatic perfusion [8], resulting in a lower impact of occluded portal veins in patients with cirrhosis. In TARE, where hepatic arteries are occluded and not portal veins, cirrhosis seems to be a minor factor for hypertrophy. This result is in keeping with a recent review by Birgin et al., which showed no significant difference of kinetic growth rate after TARE in patients with and without cirrhosis [17].

In summary, in our study, there was similar hypertrophy of the contralateral liver lobe in patients with and without cirrhosis after TARE, compared to PVE, where hypertrophy in patients without cirrhosis was more pronounced. Thus, cirrhosis seems to be less of a limiting factor for hypertrophy after TARE. Nevertheless, in the complete subgroup of patients with cirrhosis, hypertrophy after PVE was more pronounced than after TARE. Since TARE offers the added potential benefit of local tumor control and downsizing, further prospective studies are necessary to evaluate if TARE could be an alternative to PVE in patients with cirrhosis.

One of the main reasons for the implementation of this study was the known high variability in hypertrophy and the evaluation of possible influencing factors, which are not yet well studied. We showed that cirrhosis is one relevant factor, but there is still a high variability in hypertrophy in each examined subgroup of this study. Hypertrophy ranges from slight increase up to over $90 \%$ relative increase in volume. Furthermore, some patients showed decreased volume of the FLR/contralateral liver lobe after PVE/TARE. In the most pronounced case, the FLR shrunk by about $30 \%$ after PVE. This patient had cholestasis and therefore likely liver edema in the CT examination before PVE, then received percutaneous transhepatic biliary drainage, and (without repeat CT/ MRI examination) PVE was performed. On the follow-up CT scan after PVE, no cholestasis was present, which probably led to the marked decrease in liver volume. In some patients with cirrhosis, we encountered less pronounced shrinking of the contralateral liver lobe after PVE and after TARE. A possible explanation are on average longer time intervals between intervention and follow-up CT scan, which could have led to progression of cirrhosis. The majority of these cases also showed shrinking of the contralateral liver lobe below $10 \%$, which could partially also be due to inaccuracies on volumetry.

Cirrhosis seems to be one relevant influencing factor, but this study indicates that hypertrophy induction is complex and likely dependent on a multitude of factors. Several studies showed that the combination of portal venous embolization and transarterial chemoembolization, sequential or at the same time, can lead to resectability also in patients with large unilobar tumor burden [28]. Another advantage of this method seems to be longer overall and recurrence-free survival in patients with HCC [29]. To evaluate additional aspects, e.g., anatomy of portal veins or choice of embolization agents, further studies will be necessary.

The evaluation of cirrhosis in CT/MRI is a limitation of this study. Liver biopsy, which is the gold standard for diagnosing cirrhosis [30], was not routinely performed in our patient group. However, by using the above-mentioned criteria, we were able to reach substantial interrater agreement for evaluating cirrhosis in CT/MRI. Intraobserver reliability for evaluation of cirrhosis was not performed, which is another limitation of this study. The time interval between intervention and postinterventional CT/MRI after TARE was significantly longer than after PVE. Post-interventional imaging after PVE was performed prior to surgery (median 33 days), whereas imaging after TARE followed routine protocols (median 52.5 days). This renders sufficient matching unfeasible. To evaluate the time course of hypertrophy after TARE and PVE, further studies are necessary. Further 
limitations are the retrospective study design and the relatively low case numbers.

In conclusion, TARE induces less pronounced hypertrophy of the FLR compared to PVE. Cirrhosis seems to be less of a limiting factor for hypertrophy after TARE, compared to PVE.

\begin{abstract}
Author contributions HN: Conceptualization, Data curation, Formal analysis and investigation, Methodology, Project administration, Supervision, Writing — original draft preparation. TW: Data curation. SB: Data curation. CGR: Methodology, Writing-review and editing. VP: Methodology, Supervision, Writing - review and editing. SGS: Methodology. CR: Methodology. RTH: Project administration, Supervision, Writing-review \& editing. JPK: Conceptualization, Methodology, Project administration, Supervision, Writing—review \& editing.
\end{abstract}

Funding Open Access funding enabled and organized by Projekt DEAL.

\section{Declarations}

Ethical approval Institutional Review Board approval was obtained. Written informed consent was waived by the Institutional Review Board.

Open Access This article is licensed under a Creative Commons Attribution 4.0 International License, which permits use, sharing, adaptation, distribution and reproduction in any medium or format, as long as you give appropriate credit to the original author(s) and the source, provide a link to the Creative Commons licence, and indicate if changes were made. The images or other third party material in this article are included in the article's Creative Commons licence, unless indicated otherwise in a credit line to the material. If material is not included in the article's Creative Commons licence and your intended use is not permitted by statutory regulation or exceeds the permitted use, you will need to obtain permission directly from the copyright holder. To view a copy of this licence, visit http://creativecommons.org/licenses/by/4.0/.

\section{References}

1. Shoup M, Gonen M, D’Angelica M, et al (2003) Volumetric analysis predicts hepatic dysfunction in patients undergoing major liver resection. J Gastrointest Surg Off J Soc Surg Aliment Tract 7:325-330. https://doi.org/10.1016/s1091-255x(02)00370-0

2. Capussotti L, Muratore A, Baracchi F, et al (2008) Portal vein ligation as an efficient method of increasing the future liver remnant volume in the surgical treatment of colorectal metastases. Arch Surg Chic Ill 1960 143:978-982; discussion 982. https:// doi.org/10.1001/archsurg.143.10.978

3. Aussilhou B, Lesurtel M, Sauvanet A, et al (2008) Right portal vein ligation is as efficient as portal vein embolization to induce hypertrophy of the left liver remnant. J Gastrointest Surg Off J Soc Surg Aliment Tract 12:297-303. https://doi.org/10.1007/ s11605-007-0410-x

4. Schlitt HJ, Hackl C, Lang SA (2017) "In-Situ Split” Liver Resection/ALPPS - Historical Development and Current Practice. Visc Med 33:408-412. https://doi.org/10.1159/000479850

5. Teo J-Y, Goh BKP (2015) Contra-lateral liver lobe hypertrophy after unilobar Y90 radioembolization: an alternative to portal vein embolization? World J Gastroenterol 21:3170-3173. https://doi. org/10.3748/wjg.v21.i11.3170

6. Garlipp B, de Baere T, Damm R, et al (2014) Left-liver hypertrophy after therapeutic right-liver radioembolization is substantial but less than after portal vein embolization. Hepatol Baltim Md 59:1864-1873. https://doi.org/10.1002/hep.26947

7. Teo J-Y, Allen JC, Ng DC, et al (2016) A systematic review of contralateral liver lobe hypertrophy after unilobar selective internal radiation therapy with Y90. HPB 18:7-12. https://doi.org/10. 1016/j.hpb.2015.07.002

8. Lautt WW (1985) Mechanism and role of intrinsic regulation of hepatic arterial blood flow: hepatic arterial buffer response. Am J Physiol-Gastrointest Liver Physiol 249:G549-G556. https://doi. org/10.1152/ajpgi.1985.249.5.G549

9. May BJ, Madoff DC (2012) Portal Vein Embolization: Rationale, Technique, and Current Application. Semin Interv Radiol 29:81-89. https://doi.org/10.1055/s-0032-1312568

10. Takahashi EA, Fleming CJ, Andrews JC (2019) Future Liver Remnant Hypertrophy after Portal Vein Embolization Is Inversely Correlated with Intrahepatic Tumor Burden. J Vasc Interv Radiol JVIR 30:435-439. https://doi.org/10.1016/j.jvir.2018.10.014

11. Yim J, Hyun D, Cho SK, et al (2019) Effect of Hyperbilirubinemia on Hepatic Hypertrophy after Portal Vein Embolization and Liver Failure after Hepatectomy in Primary Biliary Malignancy. J Vasc Interv Radiol JVIR 30:31-37. https://doi.org/10.1016/j.jvir.2018. 08.006

12. Mise Y, Passot G, Wang X, et al (2016) A Nomogram to Predict Hypertrophy of Liver Segments 2 and 3 After Right Portal Vein Embolization. J Gastrointest Surg Off J Soc Surg Aliment Tract 20:1317-1323. https://doi.org/10.1007/s11605-016-3145-8

13. Madoff DC, Hicks ME, Vauthey J-N, et al (2002) Transhepatic Portal Vein Embolization: Anatomy, Indications, and Technical Considerations. Radiogr Rev Publ Radiol Soc N Am Inc 22:10631076. https://doi.org/10.1148/radiographics.22.5.g02se161063

14. Cazejust J, Bessoud B, Colignon N, et al (2014) Hepatocellular carcinoma vascularization: From the most common to the lesser known arteries. Diagn Interv Imaging 95:27-36. https://doi.org/ 10.1016/j.diii.2013.04.015

15. Cardarelli-Leite L, Chung J, Klass D, et al (2020) Ablative Transarterial Radioembolization Improves Survival in Patients with HCC and Portal Vein Tumor Thrombus. Cardiovasc Intervent Radiol. https://doi.org/10.1007/s00270-019-02404-5

16. Edeline J, Lenoir L, Boudjema K, et al (2013) Volumetric Changes after 90Y Radioembolization for Hepatocellular Carcinoma in Cirrhosis: An Option to Portal Vein Embolization in a Preoperative Setting? Ann Surg Oncol 20:2518-2525. https://doi.org/10. 1245/s10434-013-2906-9

17. Birgin E, Rasbach E, Seyfried S, et al (2020) Contralateral Liver Hypertrophy and Oncological Outcome Following Radioembolization with 90Y-Microspheres: A Systematic Review. Cancers 12:. https://doi.org/10.3390/cancers12020294

18. Salem R, Thurston KG (2006) Radioembolization with 90 Yttrium microspheres: a state-of-the-art brachytherapy treatment for primary and secondary liver malignancies. Part 1: Technical and methodologic considerations. J Vasc Interv Radiol JVIR 17:12511278. https://doi.org/10.1097/01.RVI.0000233785.75257.9A

19. Lau W-Y, Kennedy AS, Kim YH, et al (2012) Patient selection and activity planning guide for selective internal radiotherapy with yttrium-90 resin microspheres. Int J Radiat Oncol Biol Phys 82:401-407. https://doi.org/10.1016/j.ijrobp.2010.08.015

20. Gil-Alzugaray B, Chopitea A, Iñarrairaegui M, et al (2013) Prognostic factors and prevention of radioembolization-induced liver disease. Hepatol Baltim Md 57:1078-1087. https://doi.org/10. 1002/hep.26191 
21. Harbin WP, Robert NJ, Ferrucci JT (1980) Diagnosis of cirrhosis based on regional changes in hepatic morphology: a radiological and pathological analysis. Radiology 135:273-283. https://doi. org/10.1148/radiology.135.2.7367613

22. Gupta AA, Kim DC, Krinsky GA, Lee VS (2004) CT and MRI of cirrhosis and its mimics. AJR Am J Roentgenol 183:1595-1601. https://doi.org/10.2214/ajr.183.6.01831595

23. Shapiro SS, Wilk MB (1965) An Analysis of Variance Test for Normality (Complete Samples). Biometrika 52:591-611. https:// doi.org/10.2307/2333709

24. Wilcox R (2012) Introduction to Robust Estimation and Hypothesis Testing. Academic Press, Boston

25. Cohen J (1968) Weighted kappa: nominal scale agreement with provision for scaled disagreement or partial credit. Psychol Bull 70:213-220

26. McHugh ML (2012) Interrater reliability: the kappa statistic. Biochem Medica 22:276-282. https://doi.org/10.11613/BM.2012.031

27. Landis JR, Koch GG (1977) The measurement of observer agreement for categorical data. Biometrics 33:159-174. https://doi.org/ $10.2307 / 2529310$
28. Ronot M, Cauchy F, Gregoli B, et al (2016) Sequential transarterial chemoembolization and portal vein embolization before resection is a valid oncological strategy for unilobar hepatocellular carcinoma regardless of the tumor burden. HPB 18:684-690. https:// doi.org/10.1016/j.hpb.2016.05.012

29. Yoo H, Kim JH, Ko G-Y, et al (2011) Sequential Transcatheter Arterial Chemoembolization and Portal Vein Embolization versus Portal Vein Embolization Only before Major Hepatectomy for Patients with Hepatocellular Carcinoma. Ann Surg Oncol 18:1251-1257. https://doi.org/10.1245/s10434-010-1423-3

30. Sharma S, Khalili K, Nguyen GC (2014) Non-invasive diagnosis of advanced fibrosis and cirrhosis. World J Gastroenterol WJG 20:16820-16830. https://doi.org/10.3748/wjg.v20.i45.16820

Publisher's Note Springer Nature remains neutral with regard to jurisdictional claims in published maps and institutional affiliations. 